\title{
Estudio de la extrusión indirecta con punzón de sección transversal cuadrada del aluminio 6061 usando el método de elementos finitos
}

\author{
ANDRÉS FELIPE ZÚÑIgA HolguÍN ${ }^{1}$ \\ CARlos AndrÉs Mesa Montoya ${ }^{1}$ \\ $\triangle$ LuIS CARLOS FlóREz GaRCÍA ${ }^{1}$
}

\section{Resumen}

En el presente trabajo se realiza el estudio del proceso de extrusión indirecta en frio del aluminio AISI 6061 mediante el método de elementos finitos. Se estudia el comportamiento de una barra de sección transversal circular sometida a extrusión indirecta con un punzón cuadrado para evaluar características del proceso variando el redondeo del punzón y el coeficiente de fricción.

Para llevar a cabo el estudio, se realizaron investigaciones acerca del proceso de conformado de la extrusión indirecta con las cuales se planteó el objeto descrito anteriormente, el cual fue desarrollado utilizando el método de elementos finitos mostrando como resultados que la carga de la extrusión indirecta es mayor a medida que el punzón realiza su recorrido. Además, se concluye que el comportamiento del esfuerzo equivalente de Von Mies en el tocho de trabajo se mantiene constante alrededor de la cabeza del punzón a medida que este penetra el tocho.

Palabras clave: Coeficiente de fricción, Extrusión indirecta, Método de elementos finitos, Redondeo del punzón.

1 Universidad Tecnológica de Pereira. Pereira, Colombia.

Autor de correspondencia: Flórez García, L.C. (Luis Carlos): Carrera 27 \#10-02 Barrio Alamos - Risaralda - Colombia - AA: 97. Teléfono: 057 - 3105064840.

Correo electrónico: louis@utp.edu.co
Historia del artículo:

Artículo recibido: 2-XI-2018/ Aprobado: 30-V-2019

Disponible online: 3 de julio de 2019

Discusión abierta hasta marzo de 2021 


\title{
Study of the indirect extrusion with a square transverse section punch of the aluminum 6061 using the finite element method
}

\begin{abstract}
In this paper, the three-dimensional simulation of the indirect cold extrusion of aluminum is made, in which the structural static module of the ANSYS program is used. The behavior of a circular cross-section bar subjected to indirect extrusion with a square punch is studied to evaluate the characteristics of the process, varying the roundness of the punch and the coefficient of friction.

To carry out the study, investigations were made about the process of forming the indirect extrusion with which the object described above was raised, which was developed using the finite element method showing as results that the indirect extrusion load is greater as the punch travels. In addition, it is concluded that the behavior of the equivalent effort of Von Mies in the work billet remains constant around the head of the punch as it penetrates the billet.
\end{abstract}

Keywords: Backward extrusion, Finite element method, Friction coefficient, Punch rounding.

\section{Estudo da extrusão indireta com punção de seção transversal quadrada do alumínio 6061 utilizando o método dos elementos finitos}

\section{Resumo}

Neste trabalho, é feita a simulação tridimensional da extrusão a frio indireta de alumínio, na qual é utilizado o módulo estrutural estático do programa ANSYS. 0 comportamento de uma barra circular de seção transversal submetida à extrusão indireta com punção quadrada é estudado para avaliar as características do processo, variando a redondeza do punção e o coeficiente de atrito.

Para realizar o estudo, foram feitas investigações sobre o processo de formação da extrusão indireta com a qual o objeto descrito acima foi levantado, o qual foi desenvolvido utilizando o método dos elementos finitos mostrando como resultado que a carga indireta de extrusão é maior à medida que o punção viaja. Além disso, conclui-se que o comportamento do esforço equivalente de Von Mies no tarugo de trabalho permanece constante em torno da cabeça do punção ao penetrar no tarugo.

Palavras-chave: Arredondamento de punção, Coeficiente de fricção, Extrusão indireta, Método dos Elementos Finitos. 


\section{Introducción}

La extrusión es un proceso de formado por compresión en el cual el metal de trabajo es forzado a fluir a través de la abertura de un troquel para darle forma a su sección transversal (P.Groover, 2007). En la extrusión indirecta, también llamada extrusión hacia atrás y extrusión inversa, ver Figura 1, al penetrar el punzón en la pieza de trabajo, fuerza al metal a fluir a través del claro en una dirección opuesta a la del punzón (P.Groover, 2007).

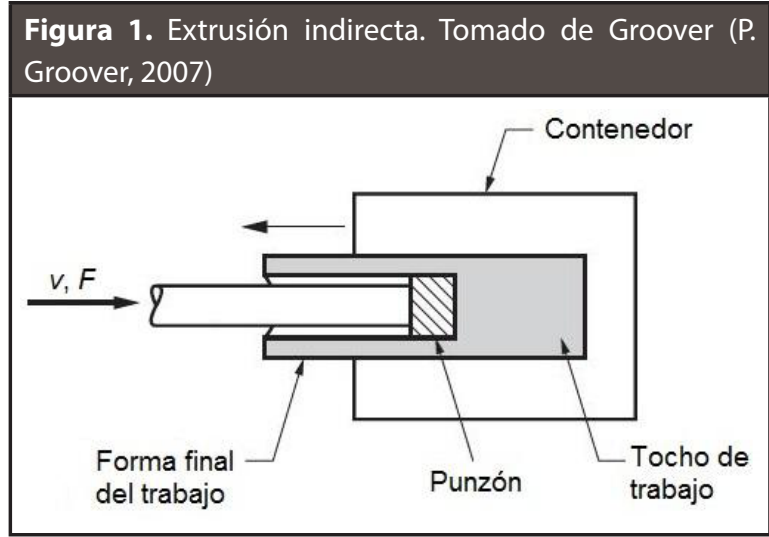

La aplicación industrial de procesos de conformado de metales tales como la extrusión indirecta han encontrado muchas aplicaciones prácticas en la producción de piezas de automóviles y aviones (Lee and Kwan, 1996).

Una de las tendencias significativas en la investigación y la aplicación de la extrusión en frío es el aumento de la complejidad de la pieza de trabajo (Bae, 1992), (Bae, 1993), (Kim, Chung and Padmanaban, 2006). Esto se puede lograr combinando los procesos de extrusión básica tales como directa, indirecta, hidrostática y extrusión por impacto, los cuales conducen a un flujo de material complejo dentro de la matriz. Como resultado de estos procesos se puede obtener un espectro más amplio de geometrías de componentes (Farhoumand and Ebrahimi, 2009). Los análisis teóricos de los tipos básicos de extrusión en frío están bien establecidos, todo lo contrario a las combinaciones de los diferentes tipos de extrusión (Plan et al., 2012). La extrusión en frío es uno de los procesos más eficientes en la tecnología de la plasticidad, tiene la característica de contar con un consumo mínimo de material y propiedades mecánicas mejoradas del componente extruido (Plan et al., 2012).

K. Abrinia (Abrinia and Orangi, 2009; Abrinia and Orangi, 2010) realiza la extrusión indirecta con diferentes formas de punzón estudiando los valores de esfuerzo, deformación y variación de fuerza con el cambio de coeficiente de fricción. Por otro lado, Gow-Yi Tzou (Tzou, Hsu and Kuo, 2012) estudia los efectos del factor de fricción, ángulo de la cara del punzón y longitud de agarre de este, analizando la influencia de estas frente a la fuerza aplicada. En (Figueroa Pilz et al., 2010) se analiza por el método de elementos finitos (MEF) el coeficiente de fricción, la altura de la matriz y la geometría del punzón sobre la carga de la extrusión. El análisis de lubricantes sólidos para el proceso de conformado lo realizan en el estudio sobre (Kim, Chung and Padmanaban, 2006), concluyendo que el coeficiente de fricción tiene un efecto importante en la carga de formación.

En el presente trabajo se plantea el uso de diferentes redondeos en la punta del punzón de sección cuadrada considerando el efecto del coeficiente de fricción entre las superficies en contacto, con el fin de estudiar la influencia de estas variables sobre la fuerza de extrusión, el esfuerzo y la deformación plástica total en el tocho de trabajo. Lo anterior busca ampliar los conocimientos generados en los estudios de extrusión indirecta en frio planteados por Plancak (Plancak et al., 2012; Plancak, 2012), García (García-Domínguez et al., 2015) y Long (Long, 2006).

\section{Extrusión indirecta en frío}

La extrusión indirecta en frío es un procedimiento de conformado plástico industrial donde se coloca en el contenedor un material de trabajo, se empieza a someter a presión por medio de un punzón. Cuando la solicitación en la pieza alcanza el límite de fluencia, el tocho de trabajo es desplazado hacia la dirección donde no se presente resistencia 
alguna, siendo opuesto al sentido de desplazamiento del punzón (García et al., 1990).

En general, se usa para producir barras cilíndricas o tubos huecos, pero se pueden producir formas de sección transversal irregular a partir de los materiales con bajo esfuerzo de fluencia (Dieter and Bacon, 1988). Dentro de las variables más estudiadas se encuentra el flujo del material, la distribución del esfuerzo y la tensión, la influencia de los parámetros del proceso como la fricción, la reducción del área, el factor de complejidad de forma y otros parámetros en la presión de extrusión y el producto final (Orangi, Abrinia and Bihamta, 2011).

En la extrusión inversa se analiza el parámetro de relación de extrusión o relación de reducción que se define de acuerdo con Ecuación 1.

$$
r_{x}=\frac{A_{o}}{A_{f}}
$$

Donde $r_{x}$ es la relación de reducción, $A_{o}$ es el área inicial y $A_{f}$ es el área final. De acuerdo con (P.Groover, 2007), la $r_{x}$ se puede emplear para determinar la deformación real $\epsilon$, ver Ecuación 2.

$$
\epsilon=\operatorname{In}\left(r_{x}\right)=\operatorname{In}\left(\frac{A_{o}}{A_{f}}\right)
$$

La presión en el punzón $p$ se puede estimar con la fórmula de Johnson (Ecuación 3) y a partir de esta, la fuerza de extrusión (Ecuación 4).

$$
\begin{aligned}
& p=S_{f} \bullet \epsilon \\
& F=A_{o} \bullet p
\end{aligned}
$$

Donde $S_{f}$ es el esfuerzo de fluencia del material.

\section{Modelado}

\subsection{Descripción del modelo geométrico}

El modelo para la extrusión indirecta consta de una pieza cilíndrica como material de trabajo (tocho) de $28 \mathrm{~mm}$ de diámetro y $28 \mathrm{~mm}$ de altura, como se plantea en la Figura 2, donde el lado izquierdo muestra el tocho sin deformar y a la derecha se encuentra el tocho deformado después del proceso de extrusión inversa. El punzón es de sección transversal cuadrada de $12,62 \mathrm{~mm} \times 12,62 \mathrm{~mm}$ y se considera como cuerpo rígido, al igual que el contenedor, despreciando así cualquier deformación o esfuerzo que estos cuerpos puedan presentar. Las dimensiones de los elementos son basadas en los estudios realizados por (Plancak et al., 2012; Plancak, 2012).

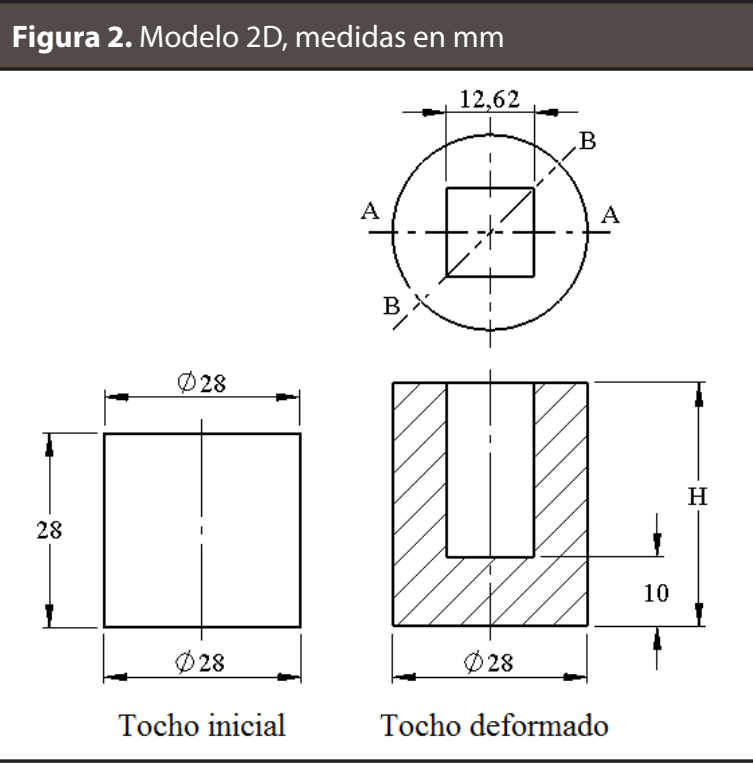

Figura 3. Modelo 3D con los componentes a modelar

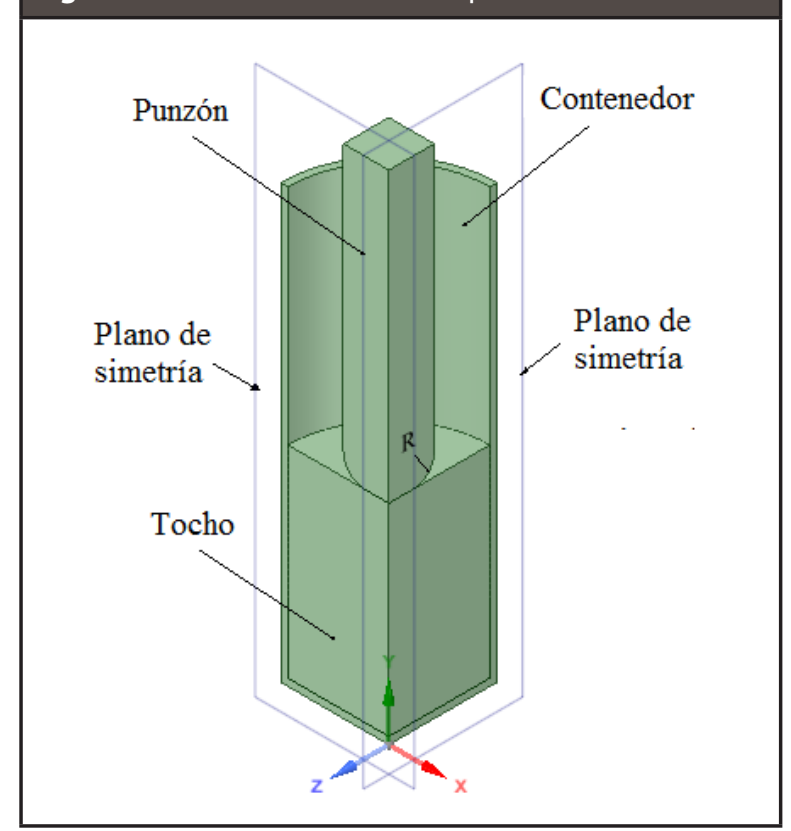


En la Figura 3 se puede ver el modelo en 3D y los planos de simetría utilizados en las simulaciones, donde $R$ es el redondeo del punzón a analizar, el cual tomará diferentes valores en los modelos. El comportamiento simétrico analizado en los modelos reduce el tiempo computacional de procesamiento para la solución de cada simulación.

\subsection{Mallado del modelo}

El modelo de elementos finitos de la pieza a trabajar usa un mallado de elementos de tipo tetraédricos vinculado con la función de región adaptativa no lineal, esta función divide los elementos tetraédricos de la malla que presentan deformaciones en la oblicuidad (skewness) menores a 0,5 y radio Jacobiano mayores a 0,1 generando nuevos elementos con tamaños menores a los elementos iniciales, con valores de simetría y radio Jacobiano dentro de los criterios establecidos.

El modelo inicia con 2019 elementos y una calidad de promedio de malla de 0,76594; debido a la función de región adaptativa no lineal la simulación realiza 83 divisiones de malla a lo largo del procesamiento del MEF finalizando con 1039400 elementos y una calidad promedio de malla en el tocho deformado de 0,8086. En la Figura 4 se observa el cambio de malla.

Figura 4. Mallado inicial y mallado final del modelo

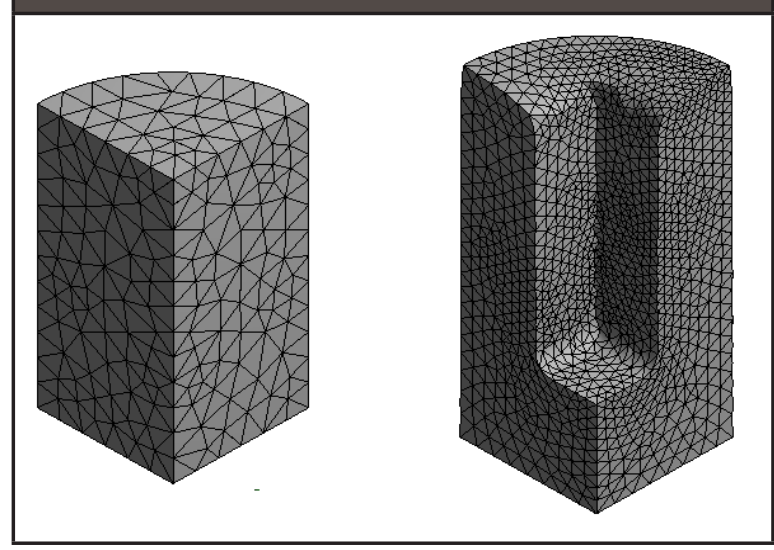

Cabe destacar que los elementos que no han sufrido deformaciones mayores a los criterios es- tablecidos se mantienen y no son divididos por la función de región adaptativa.

Debido al uso de la función de región adaptativa, la independencia de malla muestra un resultado de la fuerza máxima resultante del punzón para realizar la extrusión indirecta vs la cantidad de elementos iniciales en el mallado, presentándose una variación 0,38 \%, de acuerdo con la Figura 5. Las simulaciones presentadas en este trabajo, inician con un tamaño de elemento de malla de 3,5981 $\mathrm{mm}$ por ser el valor que consume menor tiempo computacional y presenta mayor número de divisiones de elementos.

Figura 5. Independencia de malla basado en el punzón con redondeo de $1,5 \mathrm{~mm}$

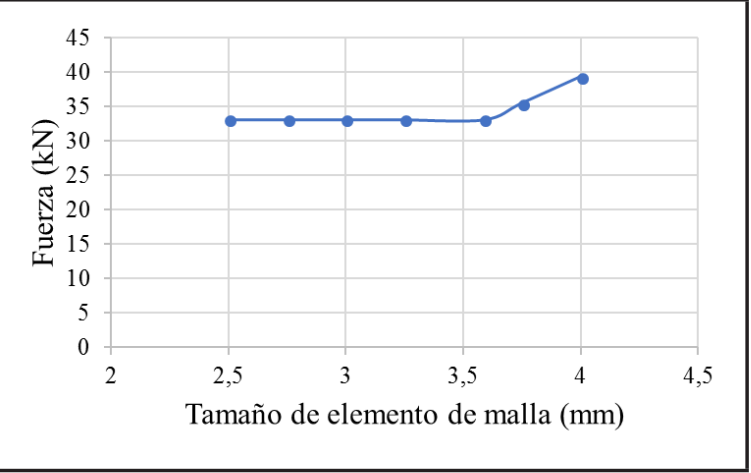

\subsection{Caracterización del material}

La caracterización del material utilizado en la simulación se realiza por medio de ensayos de tracción a diferentes probetas de aluminio 6061 de la misma procedencia, con el fin de encontrar las propiedades mecánicas necesarias para la simulación. Los ensayos consisten en someter una probeta a carga axial hasta la rotura registrando la deformación y la carga; las características buscadas son el módulo de Young, esfuerzo de fluencia y esfuerzo de rotura. Las probetas utilizadas se fabricaron con las dimensiones bajo el estándar ASTM E8.

A partir de los ensayos realizados, se construye la curva esfuerzo vs deformación real y la de ingeniería (ver Figura 6), los cuales muestran el comportamiento elástico y plástico del aluminio a trabajar. 
Figura 6. Curva esfuerzo vs deformación del material real e ingenieril

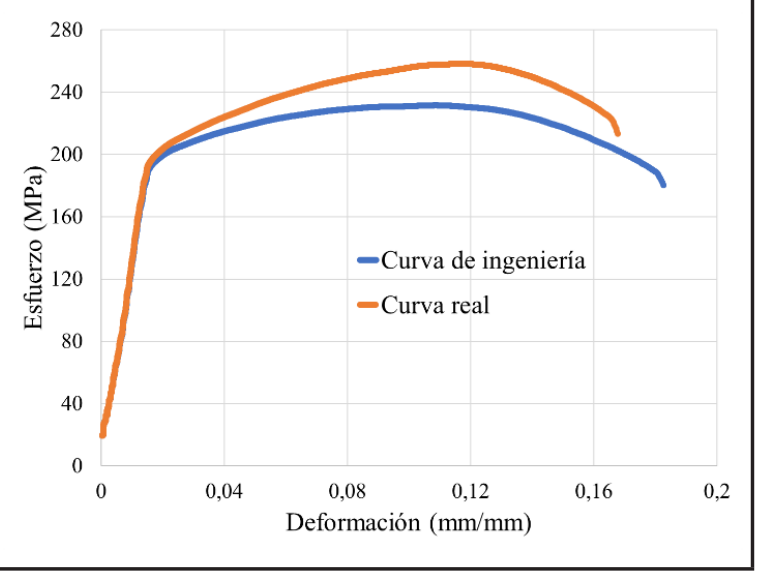

Con los datos obtenidos en los ensayos mecánicos, mediante el software ANSYS se determinan los cinco términos asociados al modelo de endurecimiento cinemático de Chaboche (Besson et al., 2010), (ver Tabla 1), los cuales se utilizan para definir el comportamiento del material en el software.

\section{TABLA 1. COEFICIENTES DE CHABOCHE}

\begin{tabular}{c|c|c|c|} 
C (MPa) & Valor & G (-) & Valor \\
\hline C1 & 506,25 & G1 & 16,68 \\
\hline C2 & 598,12 & G2 & 121,01 \\
\hline C3 & 506,42 & G3 & 16,83 \\
\hline C4 & 708,66 & G4 & 121,04 \\
\hline C5 & 506,41 & G5 & 16,55 \\
\hline
\end{tabular}

Las propiedades mecánicas del aluminio trabajado se pueden observar en la Tabla 2 .

\section{TABLA 2. PROPIEDADES MECÁNICAS DEL MATERIAL}

\begin{tabular}{c|c|c|c|c|}
\hline Material & $\begin{array}{c}\text { Esfuerzo } \\
\text { Rotura }\end{array}$ & $\begin{array}{c}\text { Esfuerzo } \\
\text { Fluencia }\end{array}$ & $\begin{array}{c}\text { Módulo } \\
\text { Young }\end{array}$ & $\begin{array}{c}\text { Relación } \\
\text { Poisson }\end{array}$ \\
\hline Unidad & $\mathrm{MPa}$ & $\mathrm{MPa}$ & $\mathrm{GPa}$ & - \\
\hline $\begin{array}{c}\text { Aluminio } \\
6061\end{array}$ & 257,42 & 173,27 & 59,15 & 0,33 \\
\hline
\end{tabular}

El modelo se utiliza debido a la aproximación que presenta con la curva real de deformación en la zona plástica, la cual se puede observar en la Figura 7.
Figura 7. Curva esfuerzo vs deformación en la zona plástica del material

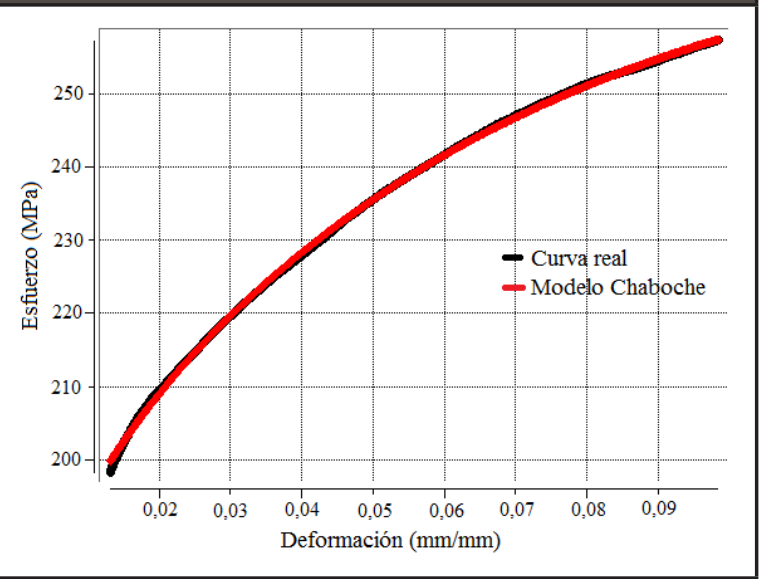

\subsection{Condiciones de frontera}

El modelo de extrusión indirecta simulado posee como condiciones de frontera los contactos que presenta el tocho con el contenedor y el punzón, estos se encuentran a lo largo de toda la superficie del tocho en diferentes caras, el contenedor entra en contacto con la base y la cara exterior del tocho, mientras que el punzón lo hace con la cara superior del material de trabajo.

Inicialmente, los contactos se consideran sin fricción con el fin de analizar la influencia del redondeo del punzón en la fuerza de extrusión Posterior a ello, se añaden valores de fricción entre todos los contactos, tanto contenedor-tocho comopunzón y se evalúa la influencia de la fricción en el proceso de conformado.

A las caras interiores del contenedor se les restringe el movimiento tanto lineal como angular en todos los sentidos para simular un comportamiento de cuerpo rígido. Por otro lado, el punzón posee un grado de libertad en el eje vertical, el cual tiene un desplazamiento de $18 \mathrm{~mm}$ en dirección del tocho, simulando el proceso de extrusión indirecta.

\section{Resultados}

La extrusión indirecta presenta las mayores cargas en el punzón al final de su recorrido, en este 
punto el tocho esta deformado en su totalidad de acuerdo con el desplazamiento planteado en la simulación. A continuación, se analiza el material de trabajo después de ser deformado bajo un coeficiente de fricción de 0 y un redondeo en el punzón de $3 \mathrm{~mm}$.

En la Figura 8 se observa el comportamiento del material extruido presentando un esfuerzo máximo en la capa que está en contacto con la cara normal al desplazamiento del punzón. Este comportamiento se aprecia a lo largo del recorrido realizado por el punzón, además de ser un valor aproximadamente constante, en este caso alrededor de $275 \mathrm{MPa}$.

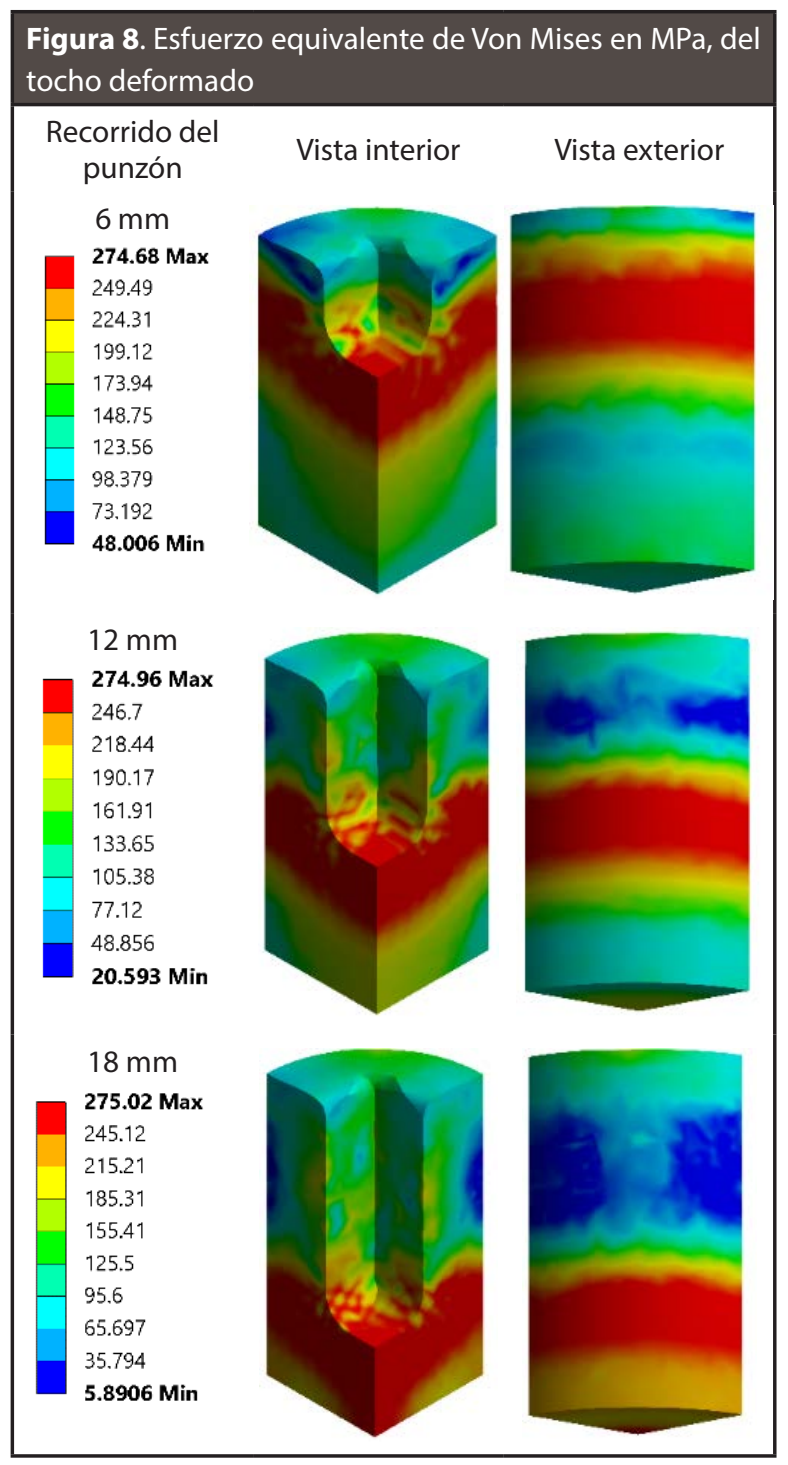

Como se puede observar en el análisis realizado en la Figura 9, el valor del esfuerzo normal al igual que el esfuerzo equivalente de Von Mises presentados en la Figura 8, mantiene un valor constante en la zona de compresión del punzón sobre el tocho. Cabe destacar en la Figura 9 que la zona de mayor esfuerzo a compresión es la zona azul, esta se mantiene a medida que el punzón penetra; además, se puede observar un comportamiento de esfuerzo a tracción del material que se está extruyendo hacia arriba donde el mayor esfuerzo se presenta en los contactos de las paredes laterales del punzón y la pared del contenedor (zona roja).

Figura 9. Esfuerzo normal en MPa, del tocho deformado

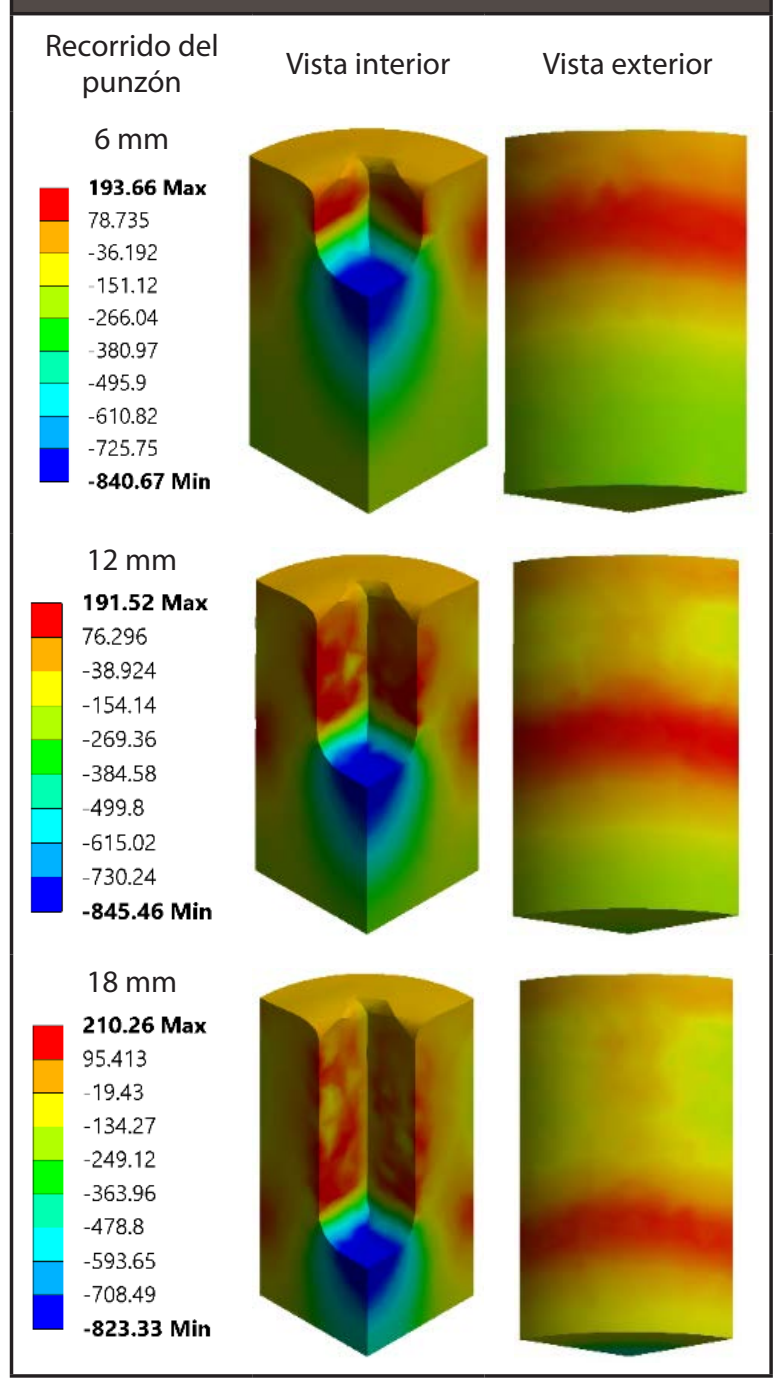


En la Figura 10 se observa el comportamiento del esfuerzo y la deformación unitaria total en el plano A-A donde la pared extruida tiene un mayor espesor, por lo tanto, se encuentra sometida a menores esfuerzos, por el contrario, la Figura 9 muestra el plano B-B donde el espesor de pared es menor mostrando esfuerzos mayores. Adicional a lo anterior, se puede confirmar lo enunciado por Plan et al en (Plan et al., 2012) que la distribución de esfuerzos y la deformación unitaria total es de la misma forma en los dos diferentes planos, con la diferencia que en la pared más delgada estos son mayores.

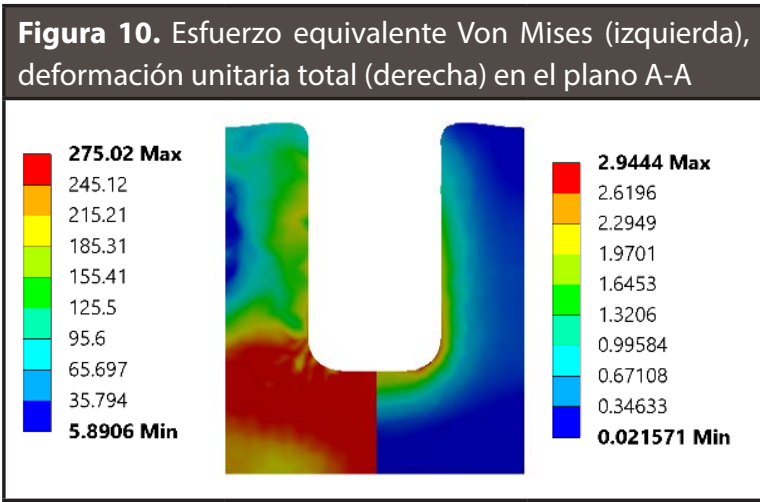

La Figura 11 muestra la deformación unitaria total del material extruido evidenciando que los puntos de mayor deformación de material son los que se encuentran en las aristas que están normal a la trayectoria del punzón, sobre todo en el vértice de este.

\section{Figura 11. Deformación unitaria total}
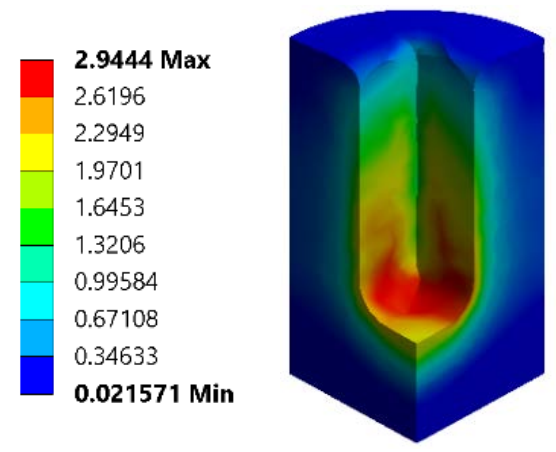

En la Figura 12 se logra observar el comportamiento de la fuerza de extrusión en el punzón vs desplazamiento punzón, donde la carga de forma- ción aumenta de manera brusca al inicio del recorrido del punzón y luego crece de manera gradual hasta el final del proceso, evidenciando que este es el comportamiento típico mostrado por diferentes autores (P.Groover, 2007; (Plancak et al., 2012; García-Domínguez et al., 2015; Dieter and Bacon, 1988); también se aprecia la influencia de la fricción en la extrusión indirecta, a medida que incrementa el coeficiente de fricción se requiere mayor fuerza para realizar el recorrido.

\section{Figura 12. Fuerza vs recorrido del punzón}

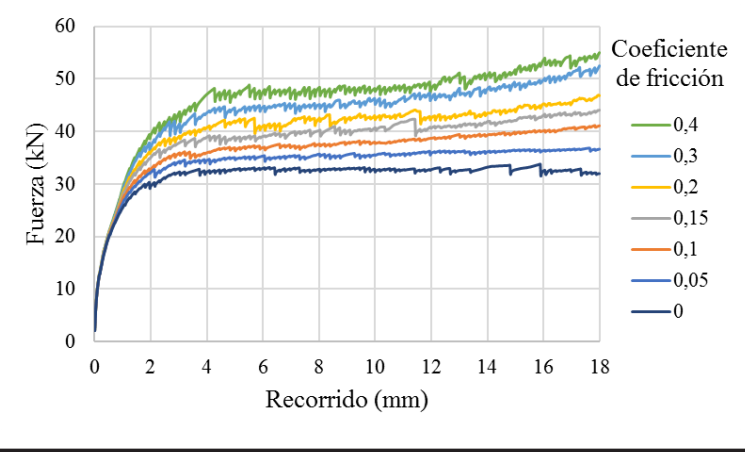

La Figura 13 muestra el comportamiento de la fuerza máxima de extrusión conforme, se varía el radio de redondeo del punzón, donde se observa que a medida que el punzón tiene un redondeo mayor, la fuerza de extrusión disminuye. Por otro lado, los cálculos de la fuerza teórica a partir de la fórmula de Johnson (P.Groover, 2007) presentan una variación entre el $0 \%$ y el $5 \%$ comparado con los diferentes valores de redondeo sin involucrar la fricción.

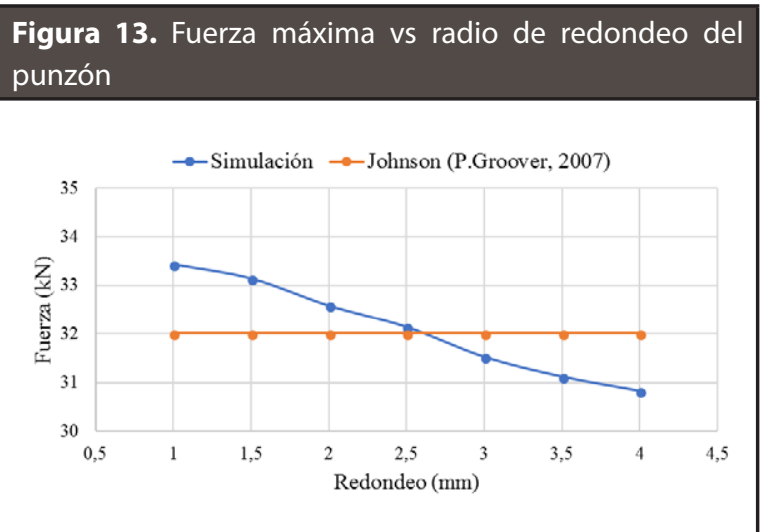


En la Figura 14, se observa que el esfuerzo equivalente máximo Von Mises en el tocho al final del recorrido permanece constante sin importar la variación del redondeo del punzón, ni el coeficiente de fricción al que está sometido este.

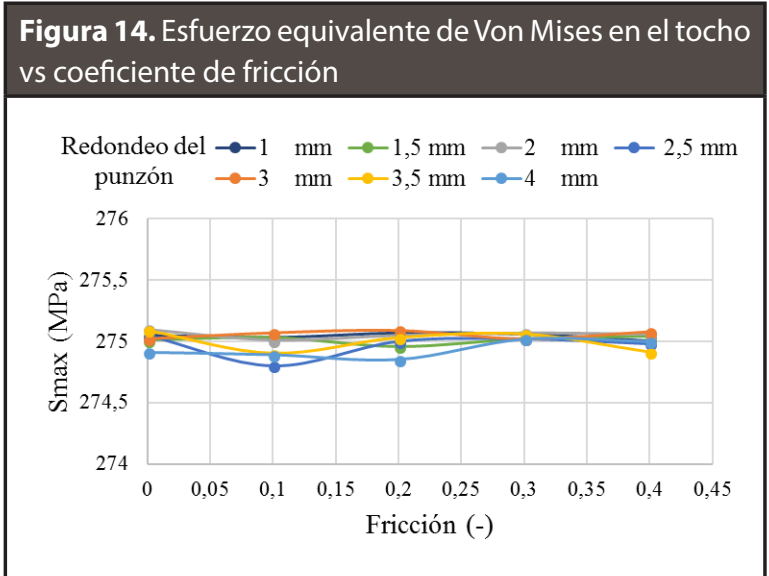

En la Figura 15, se puede observar que el valor de la fuerza máxima de extrusión es directamente proporcional al coeficiente de fricción confirmando lo afirmado por K. Abrinia (Abrinia and Orangi, 2009), donde la fuerza de extrusión aumenta conforme la fricción incrementa. De la misma forma ocurre con el incremento de fuerza y el aumento del redondeo, a medida que el redondeo aumenta junto a la fricción, la fuerza máxima es mayor.

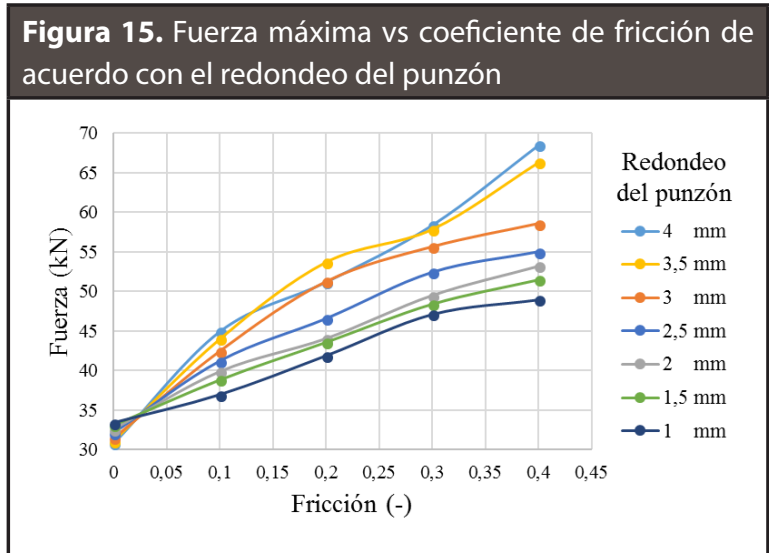

\section{Conclusiones}

En este trabajo se presenta un modelo de elementos finitos de extrusión indirecta de alumi- nio donde se varían parámetros como el radio de redondeo y coeficiente de fricción. A partir de las simulaciones realizadas se observa que la fuerza de extrusión es directamente proporcional al coeficiente de fricción.

El comportamiento del esfuerzo equivalente de Von Mies en el tocho de trabajo se mantiene constante alrededor de la cabeza del punzón a medida que este penetra el tocho.

Se logra ver que la fórmula de Johnson para calcular la fuerza de extrusión se aproxima a los valores simulados arrojando diferencias de hasta el 5 \% cuando no se está considerando la fricción,

El valor del esfuerzo máximo equivalente de von Mises en la extrusión indirecta es independiente del radio de redondeo y el coeficiente de fricción según los resultados obtenidos en las simulaciones.

En un futuro se recomienda estudiar el comportamiento de las mismas variables, teniendo en cuenta diferentes tamaños y formas de punzón.

\section{Referencias}

Abrinia, K. and Orangi, S. (2009) 'Investigation of process parameters for the backward extrusion of arbitraryshaped tubes from round billets using finite element analysis', Journal of Materials Engineering and Performance, 18(9), pp. 1201-1208. doi: 10.1007/ s11665-009-9364-3.

Abrinia, K. and Orangi, S. (2010) 'Numerical study of backward extrusion process using finite element method', Finite Element Analysis, (August), pp. 381407. doi: 10.5772/10219.

Bae, W. B. (1992) 'An upper-bound analysis of the backward extrusion of internally elliptic-shaped tubes from round billets', 30, pp. 13-30.

Bae, W. B. (1993) 'An upper-bound analysis of the backward extrusion of tubes of complicated internal shapes from round billets', 36, pp. 157-173.

Besson, J. et al. (2010) Non-Linear Mechanics of Materials, Solid Mechanics and its Applications. doi: 10.1007/978-90-481-3356-7.

Dieter, G. E. and Bacon, D. J. (1988) Mechanical metallurgy. McGraw-Hill. 
Farhoumand, A. and Ebrahimi, R. (2009) 'Analysis of forward-backward-radial extrusion process', Materials and Design. Elsevier Ltd, 30(6), pp. 2152-2157. doi: 10.1016/j.matdes.2008.08.025.

Figueroa Pilz, F. et al. (2010) 'Modelado Y Análisis Del Proceso De Extrusión Inversa En Frío Mediante Paquetería De Elemento Finito', (January 2016).

García-Domínguez, A. et al. (2015) 'Comparative analysis of extrusion processes by finite element analysis', Procedia Engineering. Elsevier B.V., 100 (January), pp. 74-83. doi: 10.1016/j.proeng.2015.01.344.

García, H. F. et al. (1990) 'Automatización neumática de un taladro de banco', Informador técnico, Centro de Desarrollo Tecnológico CDT - ASTIN SENA, 42.

Kim, S. H., Chung, S. W. and Padmanaban, S. (2006) 'Investigation of lubrication effect on the backward extrusion of thin-walled rectangular aluminum case with large aspect ratio', Journal of Materials Processing Technology, 180(1-3), pp. 185-192. doi: 10.1016/j. jmatprotec.2006.06.003.

Lee, R. and Kwan, C. (1996) 'A modified analysis of the backward extrusion of internally circular-shaped tubes from arbitrarily shaped billets by the upperbound elemental technique', 0136(95), pp. 351358.

Long, H. (2006) 'Quantitative evaluation of dimensional errors of formed components in cold backward cup extrusion', Journal of Materials Processing Technology, 177(1-3), pp. 591-595. doi: 10.1016/j.jmatprotec.2006.04.079.

Orangi, S., Abrinia, K. and Bihamta, R. (2011) 'Process parameter investigations of backward extrusion for various aluminum shaped section tubes using FEM analysis', Journal of Materials Engineering and Performance, 20(1), pp. 40-47. doi: 10.1007/s11665010-9655-8.

P.Groover, M. (2007) Fundamentos de la Manufactura Moderna. McGraw-Hill.

Plancak, M. et al. (2012) 'an Analysis of Non-Axisymmetric Backward Extrusion', pp. 953-957.

Plancak, M. (2012) 'Backward Cold Extrusion of Aluminum and Steel Billets By Non-Circular Punch', pp. 179-182.

Tzou, G. Y., Hsu, C. C. and Kuo, C. T. (2012) 'FEM Simulation Comparisons of Backward Extrusion', Advanced Materials Research, 579, pp. 42-51. doi: 10.4028/www. scientific.net/AMR.579.42.

\section{PARA CITAR ESTE ARTÍCULO / TO REFERENCE THIS ARTICLE / PARA CITAR ESTE ARTIGO /}

Zúñiga Holguín, A.F.; Mesa Montoya, C.A.; Flórez García, L.C. (2019). Estudio de la extrusión indirecta con punzón de sección transversal cuadrada del aluminio 6061 usando el método de elementos finitos. Revista EIA, 16(32), Julio-Diciembre, pp. 219-228. [Online]. Disponible en: https://doi. org/10.24050/reia.v16i32.1271 